\title{
Die Bedeutung serologischer Nachweisverfahren für die Aktivitätsdiagnostik der Syphilis
}

\author{
Der 19S-IgM-FTA-Abs-Test im Vergleich mit dem Syphilis-IgM-ELISA
}

The significance of serology tests to diagnose the activity. of syphilis:

The 19S-IgM-FTA-Abs-Test compared with the Syphilis-IgM-ELISA

Helga Mäter-Böhm', Ruth Hörnle²

'Landesmedizinaluntersuchungsamt Berlin (Direktor: Dr. M. Wundschock)

'Bezirksamt Schöneberg von Berlin, Abt. Gesundheit und Umweltschutz (Leiter: H.-J. Otto)

\begin{abstract}
Zusammenfassung:
Trotz rückläufiger Trends bei den meldepflichtigen Geschlechtskrankheiten gewinnen wegen der steigenden Zahl latenter und auch asymptomatisch verlaufender Frühsyphilisfälle die Nachweisverfahren immer mehr an Bedeutung, mit denen eine sichere Aktivitätsdiagnostik betrieben werden kann. Ein neuerEnzymimmunoassay (IgM-Capture-Test) zum Nachweis Treponema pallidum-spezifischer IgM-Antikörper wurde mit den Ergebnissen des 19SIgM-FTA-Abs-Test verglichen. Für die Referenzmethode wurde die mittels Chromatographie (FPLC) gewonnene 19S-IgM-Fraktion verwandt. 108 Seren von Patienten in verschiedenen Stadien einer Syphilis wurden auf das Vorhandensein von Syphilis spezifischen IgM-Antikörpern untersucht. Hierbei ergab sich in 96,3\% der Fälle eine Übereinstimmung mit der Referenzmethode. Es werden die beiden Methoden im Vergleich dargestellt und die Probleme bei der IgM-Serodiagnostik der Syphilis erörtert. Die Bedeutung sicherer Nachweisverfahren für die Aktivitätsdiagnostik wird diskutiert.
\end{abstract}

\section{Schlüsselwörter:}

Syphilis - Treponema-pallidium-spezifische 19S-IgM-Antikörper - CaptureSyphilis-IgM-ELISA

\section{Summary:}

In spite of decreasing numbers of notifiable venereal diseases there are increasing numbers of latent and asymptomatic infections of syphilis for which we need reliable tests to define the activity of the disease. A new EIA (IgM-capture-Test) for the detection of Treponema pallidium-specific-IgMantibodies was compared with the results obtained by 19S-IgM-FTA-AbsTest. The 19S-IgM-fraction obtained by FPLC was used as the reference method. 108 sera of patients with different stages of syphilis infection were screened for syphilis specific IgM-antibodies. In $96.3 \%$ the new test corresponded to the reference method. The two methods were compared and the problems of IgM tests in syphilis were discussed. The significance of reliable tests to diagnose the activity of syphilis was presented.

\section{Keywords:}

Syphilis - Treponema pallidum-specific-19S-IgM-antibodies - capture-Syphilis-IgM-ELISA

\section{Einleitung}

Der verstärkt in den 80 er Jahren einsetzende rückläufige Trend bei den meldepflichtigen Geschlechtskrankheiten zeigte sich auch in Berlin bei den gemeldeten Neuerkrankungen an Syphilis $(1,2)$. Dieser positive Effekt ist sicher ein Ergebnis der verstärkten Prävention bei AIDS. Statistiker gehen allerdings auch von einer erheblichen Nichterfassung der Geschlechtskrankheiten aus. Das
Meldedefizit in Mitteleuropa ist je nach Untersuchung zwischen 20 und $50 \%$ anzusetzen $(3,4,5,6)$.

Angesichts steigender Zahlen in den USA $(2,6,7)$, insbesondere bei Frauen und bei der connatalen Syphilis, sollte die Syphilisdiagnostik keineswegs vernachlässigt werden.

Die Syphilis ist nach wie vor eine epidemiologisch relevante Erkrankung, deren Wiederansteigen nur durch die 


\section{Originalie}

konsequente Weiterführung der Erfassungs- und Behandlungsmaßnahmen verhindert werden kann (8).

Die klassischen klinischen Manifestationen der Syphilis sind in den letzten Jahren seltener beobachtet worden, wogegen die zufällig im Rahmen eines Laborscreenings aufgedeckte Lues latens seropositiva heute die häufigste Erscheinungsform darstellt $(6,7,9)$.

Die breite Anwendung von Antibiotika wegen anderer Indikationen in subcurativen Dosen führt bei syphilitischen Infektionen zu maskierten Verläufen, so daß die Patienten klinisch erscheinungsfrei in die Latenzphase gelangen (4). Selbst im Stadium der Frühsyphilis können bis zu $30 \%$ der Infektionen asymptomatisch verlaufen (10).

In den letzten Jahren haben daher insbesondere die Nachweisverfahren einen höheren Stellenwert erlangt, mit denen ein sicherer Nachweis von T. pallidum-spezifischen IgM-Antikörpern gelingt und Aussagen über die Aktivität und Behandlungsbedürftigkeit der Erkrankung gemacht werden können.

Die Antikörper der IgM-Klasse treten als erstes Zeichen der humoralen Immunantwort während der frühen primären Phase der Syphilis auf und sind am Ende der 2. Woche nach Infektion nachweisbar. Mit Einsetzen der IgG-Antikörpersynthese geht die Produktion der IgMAntikörper zurück (negatives Feedback).

Während die Treponemen spezifischen IgG-Antikörper unabhängig vom Verlauf der Erkrankung persistieren, besitzen die IgM-Antikörper eine enge Beziehung zur Aktivität der Erkrankung. Das Verschwinden der spezifischen IgM-Antikörper hängt vom Zeitintervall zwischen Infektion und Therapiebeginn ab und erfolgt um so schneller, je früher die Behandlung einsetzt. Es liegt zwischen 3 bis $12(24)$ Monaten $(4,6,8)$.

Bei der Aktivitätsdiagnostik der Syphilis ist dem Nachweis Treponemen spezifischer IgM-Antikörper gegenüber den Cardiolipin- oder Reagintesten wie VDRL der Vorzug zu geben. In der frühen Infektionsphase und bei langzeitig zurückliegender infektionslatenter Treponematose sind antilipoidale Antikörper nicht immer nachweisbar (11).

Andererseits können antilipoidale Antikörper über die Ausheilung der Infektion hinaus persistieren $(12,13)$.

\section{Material und Methoden}

Wir untersuchten 108 unselektierte Serumproben von Patienten in verschiedenen Stadien der Syphilis, die an die serologische Abteilung des Landesmedizinaluntersuchungsamtes Berlin mit der Frage nach Behandlungsbedürftigkeit oder zur Kontrolle einer sanierend behandelten Lues eingeschickt wurden.

Bei allen Seren wurde zunächst routinemäßig eine serologische Syphilis-Grunddiagnostik durchgeführt. Als Suchreaktion benutzten wir den TPHA (Treponema pallidum-Haemagglutinations-Assay, Mast Diagnostika, Reinfeld). Bei reaktivem Ausfall wurde der FTA-Abs-Test (Fluoreszens-Treponema-Antikörper-Absorptions-Test,
Biologische Arbeitsgemeinschaft, Lich) als Bestätigungstest und eine quantitative Cardiolipin-Reaktion mit dem VDRL-Test (Veneral-Disease-Research-Laboratory-Test, Behringwerke, Marburg) angeschlossen.

Als weiterführende Diagnostik zur Abklärung der Behandlungsbedürftigkeit wurden dann die erregerspezifischen IgM-Antikörper mit dem 19S-IgM-FTA-AbsTest und dem Freka-Syphilis-M-ELISA (Fresenius, Bad Homburg), bestimmt.

\section{S-IgM-FTA-Abs-Test}

Die Auftrennung der Seren für den 19S-IgM-FTA-AbsTest erfolgte durch Gelchromatographie. Wir führten die Trennschritte vollautomatisch mit einerFPLC-Anlage von Pharmacia (Pharmacia LKB, Uppsala, Sweden) durch. Für die Abtrennung der 19S-IgM-Serumantikörper von den 7S-IgG-Antikörpern benutzten wir eine vorgepackte Superose 12 HR 10/30 Säule (Pharmacia, LKB, Uppsala, Sweden).

Die so gewonnenen IgM-Fraktionen wurden im FTA-AbsTest unter Verwendung eines monovalenten FITC-markierten Antihumanglobulins mit $\mu$-Kettenspezifität (Sanofi Diagnostics Pasteur, Freiburg) weiter verarbeitet.

\section{Freka-Syphilis-M-Enzymimmunoassay}

Der Freka-Syphilis-M-Test (Fresenius, Bad Homburg) ist ein IgM- "Capture-Enzymimmunoassay" zum Nachweis von IgM-Antikörpern gegen Treponema pallidum im Serum. Er kombiniert Fraktionierung und Test.

Immobilisierte Kaninchen-IgG-Antikörper gegen humanes IgM ( $\mu$-kettenspezifisch) erkennen nach Inkubation mit verdünntem Patientenserum die Gesamtheit der IgMFraktion und binden diese. Nichtgebundene Serumbestandteile werden ausgewaschen.

Der Ansatz wird mit dem Treponema pallidum Antigen (Tracerreagenz) inkubiert, das sich an Treponema pallidum spezifische IgM-Antikörper bindet. Dieser Komplex wird durch biotinylierte monoklonale Treponema pallidum Antikörper und Streptavidin markierte Meerrettichperoxydase konjugiert und durch das chromogene Substrat Tetramethylbenzidin (TMB) sichtbar gemacht. Die Farbintensität ist direkt proportional zum Anteil des an die feste Phase gebundenen spezifischen Treponema pallidum IgM. Die photometrische Auswertung erfolgte mit dem Microplate reader MR 700 bei $450 \mathrm{~nm}$.

\section{Ergebnisse}

Bei 108 Seren, die mit dem 19S-IgM-FTA-Abs-Test nach chromatographischer Auftrennung der Seren in derFPLCAnlage untersucht wurden, zeigte sich im Vergleich mit dem Freka-Syphilis-M-Test in $75 \%$ eine volle Übereinstimmung. Geringe Abweichungen in grenzwertigen Bereichen fanden wir in $21,3 \%$, so daß insgesamt von einer Übereinstimmung in $96,3 \%$ ausgegangen werden kann (Tab. 1, Abb. 1).

Die Prävalenz positiver Syphilis spezifischer IgM-Antikörper betrug in unserem Patientenkollektiv $(n=108)$ je 
Tab. 1: Vergleich der Ergebnisse von 19S-IgM-FTA-Abs-Test und Freka-Syphilis-M-Test $(n=108)$.

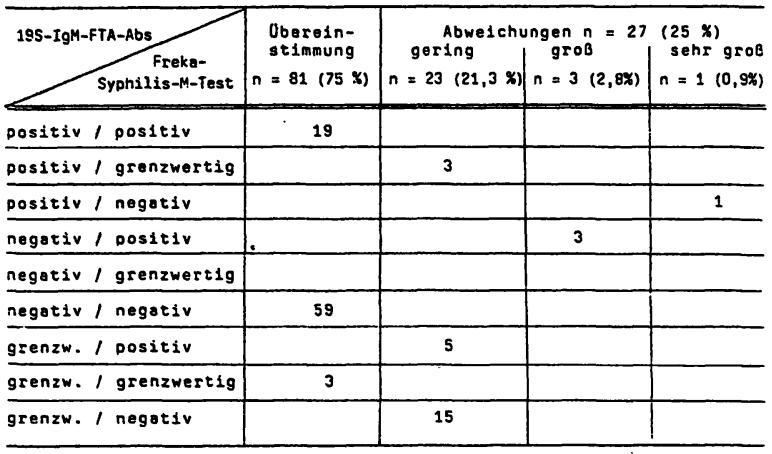

$$
\%
$$

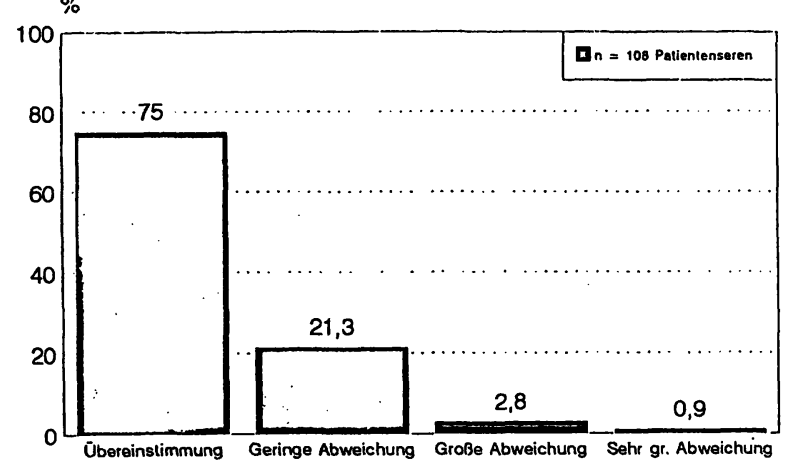

Abb. 1: Vergleich 19S-IgM-FTA-Abs-Test / Freka-Syphilis-M-Test

nach verwendeter Methode 21,3\% (19S-IgM-FTA-Abs) bzw. 25\% (Freka-Syphilis-M-ELISA).

$$
-\cdots
$$

Von den 108 Patienten in verschiedenen Stadien der Syphilis wurden mit dem 19S-IgM-FTA-Abs 23 reaktiv, 23 grenzwertig und 62 nicht reaktiv bewertet. Mit dem Syphilis-M-ELISA wurden 27 positive, 6 grenzwertige und 75 negative Ergebnisse ermittelt.

\section{Diskussion}

Die relativ hohe Zahl grenzwertiger Ergebnisse im 19SIgM-FTA-Abs-Test läßt sich in 2 Richtungen diskutieren. Die Referenzmethode besitzt bei niedrigen Antikörperkonzentrationen möglicherweise eine größere Sensitivität als der Syphilis-M-ELISA.

Andererseits unterliegen Fluoreszenztechniken auch bei erfahrenen Untersuchern aufgrund subjektiver Beurteilungskriterien geringen Schwankungen, die gerade in Grenzbereichen deutlich werden. Hier ermöglicht die IgM-Diagnostik mit Hilfe des ELISA-Testsystems ein genaueres Monitoring.

Das Ergebnis der Vergleichsuntersuchung beider Testsysteme ergab mit $96,3 \%$ Übereinstimmung ein gutes Resultat.
Große Abweichungen fanden sich nur in 3 Fällen $(2,8 \%)$. Es handelte sich dabei ausschließlich um Fälle von Lues latens seropositiva. Die TPHA-Titer lagen unter 1280. Die Referenzmethode 19S-IgM-FTA-Abs-Test war nicht reaktiv, der Syphilis-M-ELISA ergab positive Werte, die allerdings nur gering über dem Cut off lagen.

Nur in einem Fall $(0,9 \%)$ gab es eine sehr große Abweichung. Hier war 19 Monate nach Therapie der 19S-IgMFTA-Abs-Test noch-reaktiv und der Syphilis-IgM-ELISA negativ. Dabei handelte es sich um das Serum eines 1978 erstmals behandelten Patienten mit Reinfektion bei Alkoholabusus, Polytoxikomanie und verschiedenen Virusinfektionen. Damit sind weitere Kontrollen im o.g. Zeitraum (d.h. bis zu 24 Monaten) erforderlich.

Mit falsch negativen Befunden im Syphilis-IgM-EIA muß bei Reinfektionen gerechnet werden $(8,15)$. Lefevre et al (15) fanden für den IgM-EIA eine dem 19S-IgM-FTA-AbsTest vergleichbare Sensitivität bei der Primärsyphilis $(94 \%)$, die aber bei der Sekundärsyphilis $(85 \%)$, in der Frühlatenz $(82 \%)$ und bei Reinfektionen $(62 \%)$ deutlich geringer war.

Zu ähnlichen Ergebnissen kamen ljsselmuiden et al (16), die in den frühen Stadien der Syphilis mit dem IgM-EIA eine Sensitivität von $93 \%$ fanden, aber im Sekundär- und Latenzstadium sowie bei der Neurosyphilis eine verminderte Sensititivät beschrieben. In diesen Fällen wird man auf den 19S-IgM-FTA-Abs nicht verzichten können.

Nach den DGHM-Verfahrensrichtlinien (14) darf ein negatives Resultat im 19S-IgM-FTA-Abs-Test ohnehin nur dann im Sinne einer ausreichend behandelten oder spontan geheilten Syphilis gewertet werden, wenn sich aufgrund anderweitiger labordiagnostischerErgebnisse oder aufgrund fehlender klinischer Befunde kein Hinweis auf eine aktive Syphilis ergibt.

Der Syphilis-IgM-ELISA besitzt dennoch folgende Vorteile:

1. Es handelt sich um eine technisch einfach und schnell durchführbare Methode, die vollautomatisierbar ist.

2. Die apparativen Voraussetzungen sind geringer als die kostenaufwendigen FPLC- oder HPLC-Verfahren, die auch eine intensivere Einarbeitung benötigen als der ELISA.

3. Die bei der Aufarbeitung der Seren auftretenden Verluste an erregerspezifischen Antikörpern werden vermieden.

Auch wenn dieses Verfahren technisch und apparativ nicht so aufwendig ist, sollte die Syphilis spezifische IgMDiagnostik nur in größeren Laboratorien durchgeführt werden, wo die für die Interpretation der Befunde erforderlichen umfangreichen Erfahrungen vorliegen.

Nach unserer Meinung ist die Indikation zur Bestimmung -Syphilis spezifischer IgM-Antikörper weiter zu stellen, da es zu folgenschweren Fehlentscheidungen für die Patienten kommen kann, wenn die Entscheidung über die Aktivität und Behandlungsbedürftigkeit anhand eines unvollständigen Testprofils gestellt wird.

Lab.med. 18: 207 (1994) 


\section{Originalie}

Literatur:

1. Jahresgesundheitsbericht Berlin 1992 der Senatsverwaltung für Gesundheit Berlin, Referat Gesundheitsstatistik, Gesundheitsberichterstattung, Informations- und Kommunikationstechnik, Datenschutz (1993): 102-103, Berlin

2. Hörnle, R. und Suleyman, M. (1992): Geschlechtskrankheiten in Berlin. Berliner Ärzte 11, 29-31

3. Brede, H.-D. (1986): Syphilis. Münch. Med. Wschr. 128, 55-56

4. Meurer, M. und Braun-Falco, O. (1987): Latente und manifeste erworbene Syphilis - Klinik, Diagnostik und Therapie, Urologe, 26, 263-267

5. Meurer, M. und Braun-Falco, O. (1987): Klinik, Diagnostik und Therapie der Syphilis in der Schwangerschaft und bei Neugeborenen. Geburtsh, $u$. Fravenheilk. 47, 81-86

6. Gschnait, F.; Korting, H.C.; Stary, A. (1990): Sexuell übertragbare Erkrankungen. Springer, 10, 21-44

7. Gschnait, F. (1989): Ausgewählte Aspekte der Syphilisserologie, Z. f. Hautkrankheiten 64, 398-400

8. Hagedorn, H.J. (1993): Aktuelle Aspekte der Syphilisdiagnostik, Immun. Infekt. 21, 94-99

9. Smolle, J. und Turek, T.-D. (1988): Statistische Beziehungen zwischen Blutund Liquorserologie bei Syphilispatienten, Z. f. Hautkrankheiten 63 (3), 227229

10. Schmidt, B.L. ( 1990): Diagnostik von Treponema-pallidum-Infektionen des Menschen, mta 5 (3), 201-205

11. Müller, F. (1992): Methoden zum Nachweis treponemenspezifischer IgGund IgM-Antikörper - in Burkhardt, F.: Mikrobiologische Diagnostik, Thieme, Stuttgart - New York, 301-302

12. Enders, G. (1988): Infektionen und Impfungen in der Schwangerschaft; Infektionen der Mutter und des Feten; Schutzimpfungen in der Schwangerschaft, Urban und Schwarzenberg, 176

13. Schmidt, B. und Luger, A. (1979): Eine neue Methode zum serologischen Nachweis von Reinfektionen mit Treponema pallidum, Der Hautarzt, 30, 662665
14. Serodiagnostik der Syphilis (Lues). DGHM-Verfahrensrichtlinien, G. Fischer Stuttgart (8/1981)

15. Lefevre, J.-C.; Bertrand, M.-A. and Bauriaud, R. (1990): Evaluation of the Captia Enzyme Immunoassays for Detection of Immunoglobulins $\mathbf{G}$ and $M$ to Treponema pallidum in Syphilis. J. Clin. Microbiol. 28, 1704-1707

16. Ijsselmuiden, O.E.; van der Sluis, J.J.; Mulder, A.; Stolz, E.;Bolton, K.P. and van Eijk, R.V.W. (1989): An IgM Capture enzyme linked immunosorbent assay to detect IgM antibodies to treponemes in patients with syphilis. Genitourin. Med. 65, 79-83

\section{Anschriften der Verfasser:}

Dr. med. Helga Mäter-Böhm

Landesmedizinaluntersuchungsamt Berlin

Rubensstraße 111

12157 Berlin

Dr. med. Ruth Hörnle

Beratungsstelle Geschlechtskrankheiten

Bezirksamt Schöneberg von Berlin

Abt. Gesundheit und Umweltschutz

Dominicusstraße 25-27

10823 Berlin

Danksagung:

Für die ausgezeichnete technische Unterstützung bei der Durchführung der ELISA-Untersuchungen danken wir Frau Helga Garske. 


\section{[interobaterienseen!}

\section{Schbnelle Erkennumg mint dem RAM}

\section{Proteus $\rightarrow$}

\section{Salmonella $\rightarrow 0$}

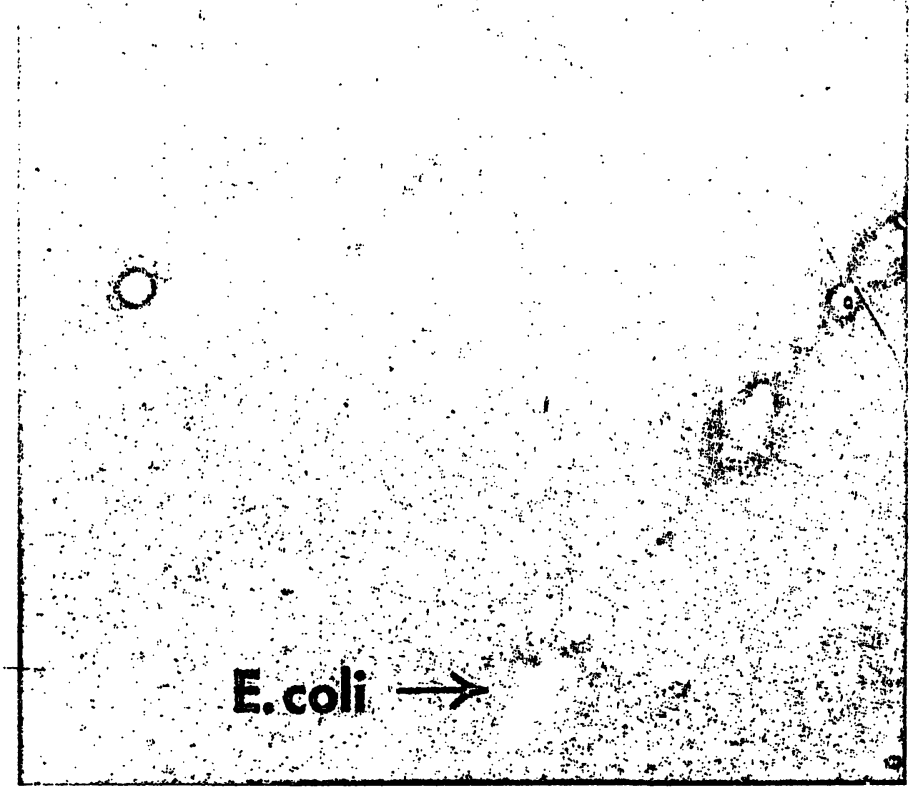

- klare Unterscheidung durch charakteristische Koloniefarbe E.coli-blau; Salmonellenrot; Proteus und Shigellen-beige

- schnell in der Regel nach 17 bis 24 Stunden

- wirtschafflich do oufwendige und teure Identifizierung von falsch pos. Kolonien entfallen

\section{- anwenderfreundlich} Trockennöhrboden fertig gemischt oder Merckoplate ${ }^{\circledR}$ Fertigplatte
Enterobacteriaceen!

Schnelle Erkennung mit dem

RAMBACH ${ }^{3}$-Agar.

\section{E. Merck - 64271 Darmstadt}

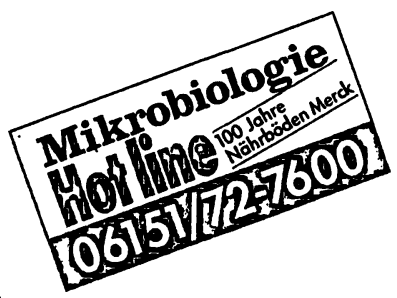

MERCK 


\section{Tabellen und Methoden}

zur medizinisch-

bakteriologischen Laborpraxis

H. Bürger, Z. Hussain Format $17 \times 24 \mathrm{~cm}$, 256 Seiten, Abbildungen, Tabellen, PVC-Einband, ISBN 3-87409-006-X, DM 68,-
Isolierung und Identifizierung pathogener Mikroorganismen sind die Voraussetzungen für Diagnose, Therapie, Verhütung von Infektionen und zur Infektionskontrolle.

In dem vorliegenden Buch werden die bisher in jedem qualifizierten mikrobiologischen Labor eingeführten kulturellen und biochemischen Verfahren beschrieben.

Die wichtigsten Daten von ca. 400 als Krankheitserreger geltenden oder aus differentialdiagnostischen Gründen im Bereich der Humanmedizin interessierenden Bakterienspezies sind in einem kompakten Abriß zusammengefaßt.

Der erste Teil des Buches informiert über Gewinnung, Transport und Verarbeitung von Untersuchungsmaterialien, der Hauptteil enthält sehr ausführlich kommentierte Tabellen zur Identifizierung der Mikroorganismen, und im Anschluß daran werden die im Text erwähnten Methoden unter Angabe von Bezugsquellen für notwendige Hilfsmittel erläutert.

Die Gliederung in acht Bakteriengruppen èrfolgt in konventioneller Weise anhand der Morphologie und des Gramverhaltens unter
Berücksichtigung der Sauerstofftoleranz. Bei gramnegativen und grampositiven Stäbchen wird die Orientierung durch ein am Kapitelanfang positioniertes Leitschema erleichtert. Es basiert auf wenigen schnell überprüfbaren Kriterien und verweist auf die ausführlich kommentierten Tabellen. Diese werden durch die Beschreibung der Anzuchtbedingungen, der Nährmedien, Färbeverfahren und Hinweise auf Bezugsquellen für die in der Bundesrepublik erhältlichen Diagnostika ergänzt.

Die zur Bezeichnung der Mikroorganismen gewählte Nomenklatur entspricht den "Approved lists of bacterial names" und folgt den von der American Society for Microbiology herausgegebenen Angaben des "International Journal of Systematic Bacteriology“.

Das zum Gebrauch am Arbeitsplatz bestimmte Buch wendet sich an Mikrobiologen, Hygieniker, Pharmazeuten, medizinisch-technische Assistentinnen und alle diejenigen, die routinemäBig bakteriologische Untersuchungen durchführen oder sich im Praktikum auf diese Tätigkeit vorbereiten. 